\title{
Negative differential electrical resistance of a rotational organic nanomotor
}

\author{
Hatef Sadeghi, Sara Sangtarash, Qusiy Al-Galiby, Rachel Sparks, Steven Bailey* \\ and Colin J. Lambert ${ }^{*}$
}

\section{Full Research Paper}

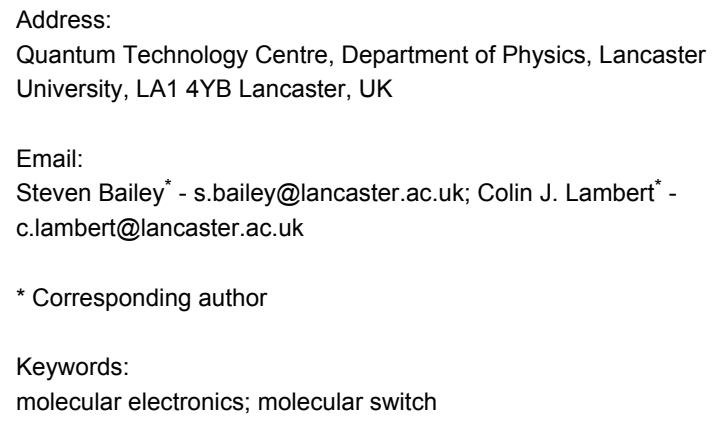

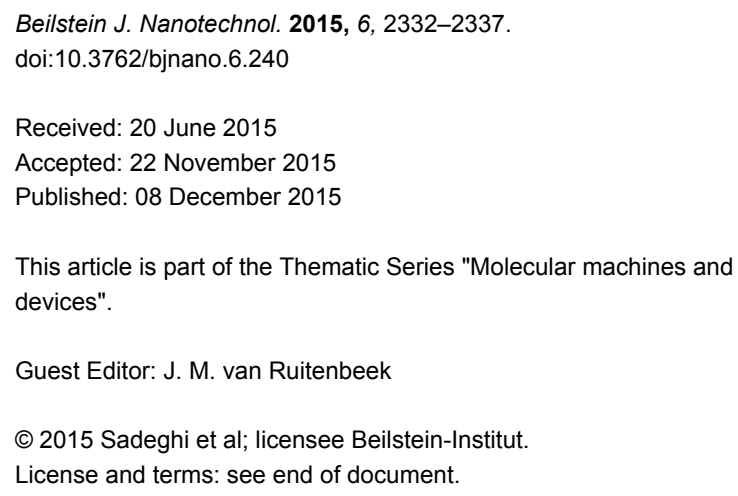

\begin{abstract}
A robust, nanoelectromechanical switch is proposed based upon an asymmetric pendant moiety anchored to an organic backbone between two $\mathrm{C}_{60}$ fullerenes, which in turn are connected to gold electrodes. Ab initio density functional calculations are used to demonstrate that an electric field induces rotation of the pendant group, leading to a nonlinear current-voltage relation. The nonlinearity is strong enough to lead to negative differential resistance at modest source-drain voltages.
\end{abstract}

\section{Introduction}

Biomotors utilising myosins, kinesins, and dyneins [1-4] have been utilised in several motor-protein-driven devices for cargo transportation [5,6], molecule sorting [7,8], imaging [9] and sensing $[10,11]$. In contrast to biological machines, which convert energy into directed motion by moving out of thermodynamic equilibrium [12-15], artificially designed nanoelectromechanical (NEM) motors operate by moving towards thermodynamic equilibrium. Many examples of artificial NEM devices use directed motion [16-26]. For example, oscillators with frequencies in excess of $1 \mathrm{GHz}$ have been constructed from multiwalled carbon nanotubes (MWCNT), where the telescoping nature of the inner carbon nanotubes $[27,28]$ with very low interwall friction [29-34] lead to novel electrical properties [35-40]. These examples illustrate how an electric field can induce motion and also how a motion-induced change of geometry can affect electrical properties. In what follows, our aim is to demonstrate that this coupling between a controlled geometry and electrical properties can lead to desirable nonlinear current-voltage relations and negative differential resistance (NDR).

As a specific example that demonstrates the general principle, we analyse the molecular-scale NEM shown in Figure 1 whose conformation can be manipulated using an external electric field 
and whose conformational changes feedback to produce a nonlinear current-voltage relation. This novel NEM consists of a pendant rotor attached by a single carbon bond to an aromatic backbone. The rotor is designed to possess a dipole moment aligned along its length such that an applied electric field will cause the rotor to turn relative to the aromatic backbone. Our aim is to examine the response of the device to an external electric field and determine the change in electrical conductance due to the associated conformational changes when the $\mathrm{C}_{60}$ molecules are attached to metallic electrodes. Our calculations will demonstrate that such conformation changes lead to NDR.

\section{Results and Discussion}

The dumbbell molecular switch shown in Figure 1 consists of three main sections, the backbone, the terminating groups and the branch. The backbone consists of five interconnected phenyl rings with attached methyl groups to prevent the backbone from twisting and is stabilized at either end by a fullerene, $\mathrm{C}_{60}$ terminating group. The $\mathrm{C}_{60}$ at either end not only stabilizes the molecule, but also allows the molecule to appear more clearly visible in STM images [41], therefore facilitating experimental STM measurements. The branch extends from the central ring of the backbone and is made up of three interlinked arene compounds; the central phenyl ring is capped by aniline at one end and terminated with a fluoro-toluene derivative at the other, where the hydrogens are replaced by fluorine. As fluorine is the most electronegative element, this design will enable the branch to possess a dipole moment. The dipole moment for the combined pendant group and backbone as an average of all the rotation angles of the pendant group relative to the backbone is approximately 9.4 debye over a length of $28.23 \AA$. The length of the pendant group alone is $20.4 \AA$. The variation in the dipole moment, over all rotation angles, is given in Supporting Information File 1, Figure S4 and the lengths are given in Supporting Information File 1, Figure S5.
This dipole moment of the branch facilitates the electric-fieldinduced rotation required to create a switch. By applying an external electric field, $E_{\text {ext }}$, across the molecule, the additional contribution to the total energy is $U=-p \cdot E_{\text {ext }}$, where $p$ is the dipole. In the presence of a uniform electric field, the energy landscape of the system will change, with the possibility that the most stable rotation angle switches from one value to another. By computing the total energy as a function of rotation angle, we thereby obtain an estimate of the size of the electric field required to switch the molecule.

We use the SIESTA [42] implementation of the density functional theory (DFT) with a van der Waals density functional $[43,44]$ and extended and corrected double-zeta-polarised basis sets of the pseudoatomic orbitals. The geometries were optimised by relaxing the atomic forces to less than $20 \mathrm{meV} / \AA$. The van der Waals density functional allows long-range interactions to be taken into account. The total ground-state energy of the molecule is calculated to find the energy profile of the molecule with different confirmations. A basis set superposition correction is carried out to account for overlapping basis functions. This correction is calculated by taking the relaxed energy of the entire molecule and subtracting the energy of the structural relaxation of backbone and the branch separately: $U_{\mathrm{BSC}}=U_{\text {molecule }}-U_{\text {branch }}-U_{\text {backbone. Figure } 2 \text { shows the }}$ potential energy profile of the dumbbell molecule against the rotation angle of the rotor with respect to the backbone. The DFT-calculated energy profile yields an energy barrier to rotation of about $800 \mathrm{meV}$.

Figure 2 shows the energy landscape as a function of rotation angle for four magnitudes of electric field between 1.0 and $3.5 \mathrm{~V} / \mathrm{nm}$. Figure $2 \mathrm{a}-\mathrm{d}$ corresponds to the directions $E^{(\mathrm{a})}, E^{(\mathrm{b})}$, $E^{(\mathrm{c})}$ and $E^{(\mathrm{d})}$, respectively, as shown in the left-most part of the figure. Only Figure $2 \mathrm{a}$ and Figure $2 \mathrm{~b}$ are relevant to the two-

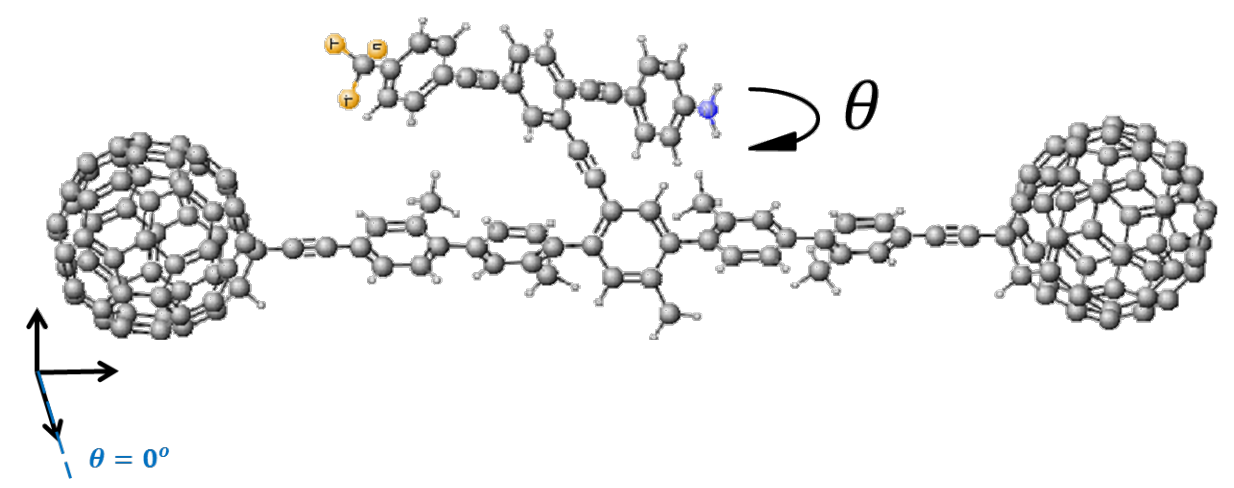

Figure 1: Schematic of the proposed molecular switch, where the asymmetric rotor blade is terminated at one end with nitrogen and at the other with three fluorine atoms with a single bond linking the rotor to the aromatic backbone. The two $\mathrm{C}_{60}$ molecules act as secure anchors for the device and could be connected to gold leads. 

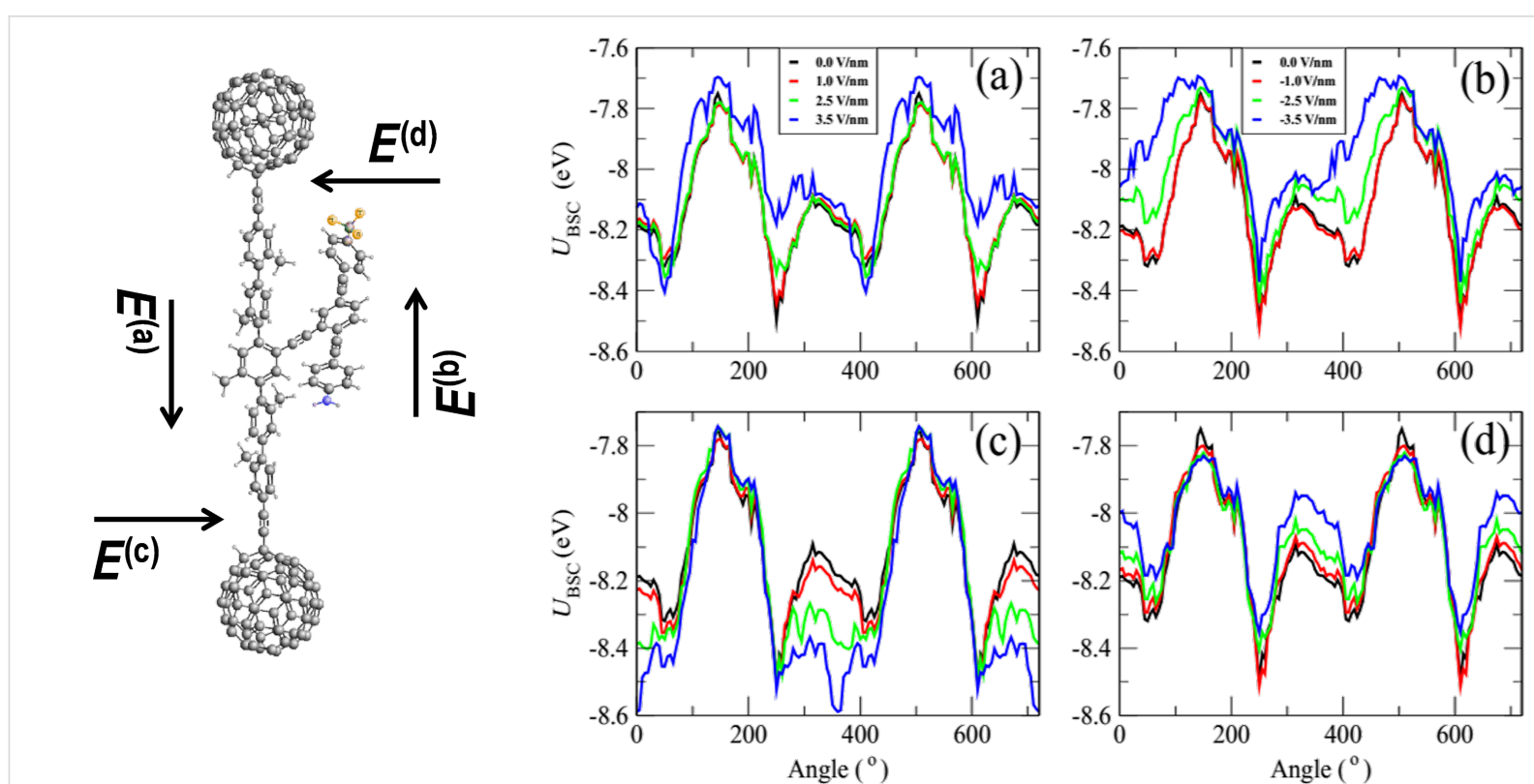

Figure 2: The potential energy profile, $U_{\mathrm{BSC}}(\mathrm{eV})$, calculated from the changes in the total energy of the system against the rotation angle of the rotor with respect to the backbone as shown in Figure 1 for four applied magnitudes of electric field between 1.0 and $3.5 \mathrm{~V} / \mathrm{nm}$. (a-d) correspond to the directions $E^{(\mathrm{a})}, E^{(\mathrm{b})}, E^{(\mathrm{c})}$ and $E^{(\mathrm{d})}$, respectively, shown in the left-most figure. The rotation angle is defined in Figure 1 and Supporting Information File 1, Figure S2. $240^{\circ}$ corresponds to the pendant group being parallel to the backbone, as shown in Figure 2 , whereas $60^{\circ}$ (or equivalently $420^{\circ}$ ) corresponds to the pendant group making a $30^{\circ}$ angle with the backbone. The zero-field energy minima are located at rotation angles of $60^{\circ}$ and $240^{\circ}$. To sample all angles and compute these energy-angle curves, the pendant group was artificially rotated to a chosen angle and then the molecule was allowed to relax to a local energy minimum.

terminal device shown in Figure 3a. The plots in Figure 2c,d are relevant to a three-terminal device containing a gate electrode able to create a field perpendicular to the length of the molecule. In what follows, we focus on a two-terminal device only, since this is likely to be realised in the laboratory. At zero temperature, the rotation angle coincides with the global minimum of the energy curves. At a finite temperature, the above minima correspond to the most-probable rotation angles, but other angles can be sampled according to the Boltzmann factor (see Equation 4 below). At zero field, the energy minima occur at $\theta_{\mathrm{A}}=60^{\circ}$ and $\theta_{\mathrm{B}}=240^{\circ}$. In both positions, the branch is parallel to the backbone and there is a significant overlap between one of the phenyl rings on the backbone with the aniline capped end of the branch. This suggests that these positions are stabilised by the $\pi-\pi$ interaction between the two aromatic rings. The rotor does not rotate at a uniform distance from the backbone and therefore the charge distribution of the branch interacts nonuniformly with the backbone. This effect is apparent at $\theta_{\mathrm{B}}$ where one end of the branch is located closer to the backbone than the other.

When an electric field is applied parallel to the backbone in the direction of $E^{(\mathrm{a})}$ (Figure 2a), the global minimum at $\theta_{\mathrm{B}}$ becomes a local minimum and the global minimum is then located at $\theta_{\mathrm{A}}$. This means that through application of an electric field parallel to the backbone, the most stable state of the molecule can be manipulated, and the branch of the molecule will switch due to this electric field. One can also observe that by removing or reversing the direction of the electric field the branch can be switched back to $\theta_{\mathrm{B}}$. As shown in Figure 2c, by applying an external field $E^{(\mathrm{c})}$ orthogonal to the molecule, the global minimum of the energy curve can be switched to $0^{\circ}$, whereas a field of $E^{(\mathrm{d})}$ causes no such crossover.

To study the effect of the external electric field on transport properties of the dumbbell molecule, consider the molecule connected to two gold electrodes in a junction, as shown in Figure 3a. Since we are interested in a two-terminal switch, we focus on the effect of the source-drain electric field induced by gold electrodes (electric field parallel to the molecule, Figure 1a,b). By contacting the left and right $\mathrm{C}_{60}$ to gold electrodes, electrons entering the leads from external reservoirs have Fermi distributions given by $f_{\mathrm{L}}(E)$ and $f_{\mathrm{E}}(E)$ and the Landauer formula [45] gives:

$$
I=\frac{2 e}{h} \int_{-\infty}^{+\infty} \mathrm{d} E T(E)\left(f_{\mathrm{L}}(E)-f_{\mathrm{R}}(E)\right)
$$

where the electronic charge $e=|e|, h$ is Plank's constant and $T(E)$ is the transmission coefficient for electrons with energy $E$ passing through the molecule from left $(\mathrm{L})$ to right $(\mathrm{R})$. 
(a)

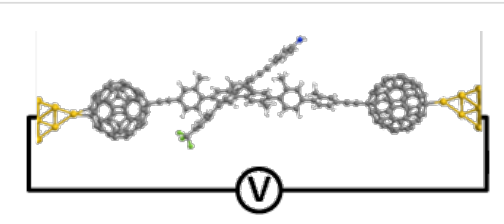

(b)

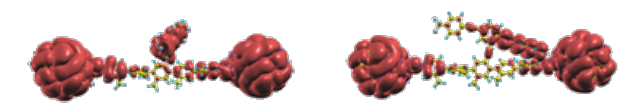

(c)

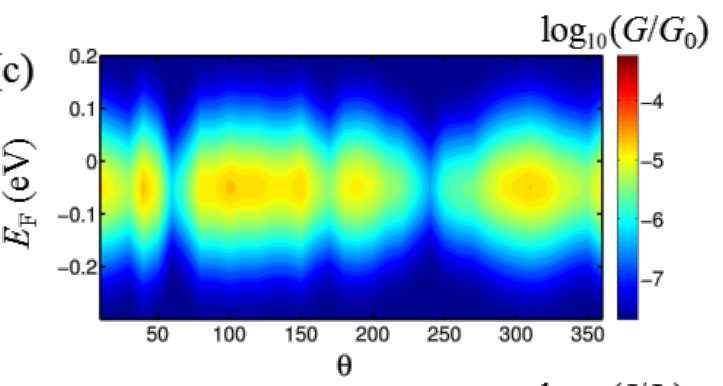

(d)

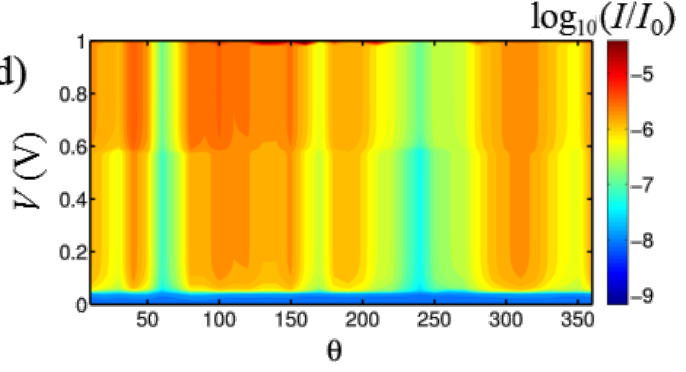

Figure 3: (a) The molecular structure within the junction. (b) A contour plot of the local density of states of the gas-phase molecule at $\theta=60^{\circ}$ (right) and $\theta=0^{\circ}$ (left). (c,d) A contour plot of the conductance $G / G_{0}$ and current $I / I_{0}\left(I_{0}=2 e^{2} / h \times 1 \mathrm{~V}=77 \mathrm{nA}\right)$, respectively, through the dumbbell molecule between two gold electrodes against angle $\theta$ degrees and $E_{\mathrm{F}}(\mathrm{eV})$ at room temperature. A contour plot of the conductance $\mathrm{G} / \mathrm{G}_{0}$ and current $\mathrm{I} / \mathrm{I}_{0}$ at zero temperature is shown in Supporting Information File 1, Figure S3.

The transmission coefficients $T(E)$ were calculated using GOLLUM [46], which is a newly developed simulation tool for electron, thermal and spin transport using the approach described in [47]. Close to equilibrium, $f_{\mathrm{L}, \mathrm{R}}(E)=\left(1+\exp \left(\mu_{\mathrm{L}, \mathrm{R}}\right)\right)^{-1}$ where $\mu_{\mathrm{L}, \mathrm{R}}=E-E_{\mathrm{F}}^{\mathrm{L}, \mathrm{R}} / k_{\mathrm{B}} T, E_{\mathrm{F}}^{\mathrm{L}}$ $\left(E_{\mathrm{F}}{ }^{\mathrm{R}}\right)$ is the Fermi energy of the left (right) reservoir and $T$ is the temperature. As shown by the transmission curves in Supporting Information File 1, Figure S1, transport is HOMOdominated. Figure $3 \mathrm{~b}$ shows contour plots of the local density of states (LDOS) around the HOMO of the isolated molecule for two different rotation angles. These plots show that the HOMO is extended, but not symmetric. This demonstrates why the transmission coefficient does not approach unity on resonance, because it is well known that the transmission coefficient is less than unity in asymmetric systems, such as the structure in Figure 1 [47-49]. At zero temperature and finite voltage $E_{\mathrm{F}}^{\mathrm{L}}=E_{\mathrm{F}}+\mathrm{eV} / 2$ and $E_{\mathrm{F}}^{\mathrm{R}}=E_{\mathrm{F}}-\mathrm{eV} / 2$ the current could be calculated as

$$
I=\frac{2 e}{h} \int_{E_{\mathrm{F}}-\mathrm{eV} / 2}^{E_{\mathrm{F}}+\mathrm{eV} / 2} \mathrm{~d} E T(E)
$$

Therefore, the electrical conductance, $G=I / V$, is obtained by averaging $T(E)$ over an energy window of width $\mathrm{eV}$ centred upon the Fermi energy. The Fermi functions can then be Taylor expanded over the range $\mathrm{eV}$ to give the electrical conductance in the zero-voltage but finite temperature limit by

$$
G=\frac{I}{V}=G_{0} \int_{-\infty}^{+\infty} \mathrm{d} E T(E)\left(-\frac{\mathrm{d} f(E)}{\mathrm{d} E}\right)
$$

which represents a thermal average of $T(E)$ over an energy window of $k_{\mathrm{B}} T$ where $k_{\mathrm{B}}$ is the Boltzmann constant. The normalised probability distribution, $-\mathrm{d} f(E) / \mathrm{d} E$, has a width of approximately $k_{\mathrm{B}} T$ so in the limit of zero voltage and zero temperature, $G=G_{0} T\left(E_{\mathrm{F}}\right)$. Figure $3 \mathrm{c}$, d shows the changes in the conductance and current, respectively, of the dumbell molecule placed between two gold electrodes for different angles between the rotor and molecular backbone. The conductance (and therefore, the current) is reduced significantly at $\theta_{\mathrm{A}}=60^{\circ}$ and $\theta_{\mathrm{B}}=240^{\circ}$ where the minima of $U_{\mathrm{BSC}}$ occur.

To include the effect of the parallel electric field due to the source-drain voltage, $V$, and induced rotation of the rotor, we use the energy landscape, $U_{\mathrm{BSC}}(\theta, V)$ to construct the probability function

$$
p(\theta, V)=A \exp \left(-U_{\mathrm{BSC}}(\theta, V) / k_{B} T\right)
$$

where $A$ is a normalisation constant. The average current at voltage $V$ can be computed using the relation:

$$
\langle I\rangle=\frac{2 e}{h} \int_{-\infty}^{\infty} \mathrm{d} E \int_{0}^{2 \pi} \mathrm{d} \theta p(\theta, V) T(E, V)\left(f_{\mathrm{L}}(E)-f_{\mathrm{R}}(E)\right)
$$

The blue curve in Figure 4 shows the weighted current at room temperature for applied biases between -1 and $1 \mathrm{~V}$. By differentiating the current with respect to the bias voltage $V$, one obtains the differential conductance (Figure 4, green dashed line) of the device, which clearly shows regions of NDR behaviour arising from the change in the energy landscape. The higher NDR effect occurs in the bias interval of $0.6-0.7 \mathrm{~V}$, although there is also a smaller NDR region at low bias voltage $\approx 0.05 \mathrm{~V}$. 


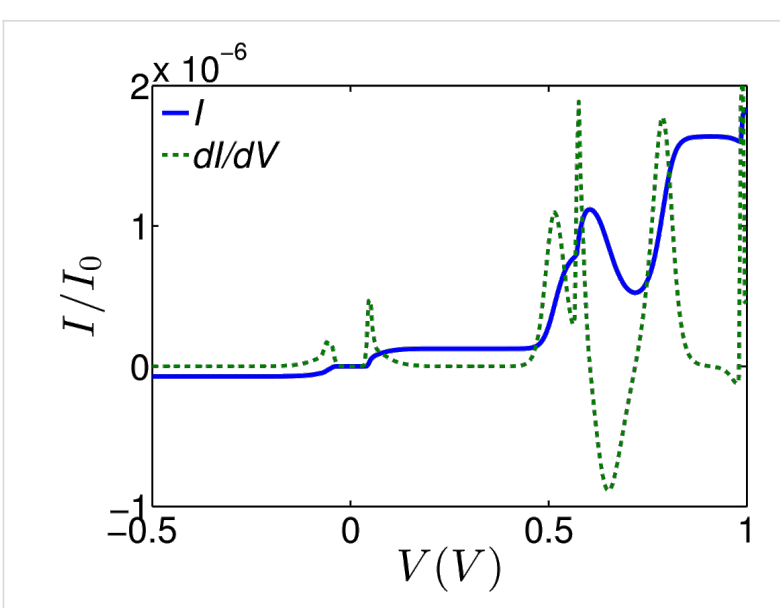

Figure 4: The weighted current (blue curve) from Equation 5 and the NDR (green curve, $\mathrm{d} / \mathrm{d} \mathrm{V}$ ) for applied bias between 0 and $1 \mathrm{~V}$. The prominent NDR features are seen at a bias voltage between $0.0-0.1 \mathrm{~V}$ and $0.6-0.7 \vee$ for the device.

At zero temperature, the rotation angle coincides with the global minimum of the energy curves. For example in Figure 2a, at zero bias (black curve), the energy minimum corresponds to an angle of $240^{\circ}$, whereas at for a field of $3.5 \mathrm{~V} / \mathrm{nm}$ parallel to the backbone (blue curve), the energy minimum corresponds to $410^{\circ}$. This demonstrates that such a field can cause the pendant group to rotate through $170^{\circ}$. On the other hand, Figure $2 \mathrm{~b}$ shows that a field in the opposite direction does not shift the global minimum and therefore does not cause the pendant group to rotate. Similarly, Figure 2c shows that a field perpendicular to the backbone can shift the minimum to $360^{\circ}$. It is these conformational changes which cause the NDR. This is because the gating of the backbone due to the dipole moment of the pendant group is angle dependent. It should be noted that the applied field does not rotate the pendant group by $360^{\circ}$. Nevertheless, the voltage-dependent energy landscape $U_{\mathrm{BSC}}(\theta, V)$ shown in Figure 2 and the associated changes in the distribution of rotation angles $p(\theta, V)$ is sufficient to produce NDR. At finite temperature, the minima in $U_{\mathrm{BSC}}(\theta, V)$ correspond to the most probable rotation angles, but other angles can be sampled according to the Boltzmann factor in Equation 4.

\section{Conclusion}

We have examined the change in conformation of a molecularscale rotator attached via a backbone to two $\mathrm{C}_{60}$ anchor groups, which in turn are connected to gold electrodes. Our aim was to determine if an applied source-drain bias could cause the equilibrium angle of the rotator to change, leading to a nonlinear current-voltage relation. Our results confirm that such a nonlinearity indeed occurs and is strong enough to lead to a pronounced negative differential resistance region at relatively low bias in the range $0.6-0.7 \mathrm{~V}$. The underlying mechanism is that the dipole moment of the pendant group electrostatically gates the backbone states and this gating is angle dependent. Such NDR behaviour is potentially of interest for molecular-scale electronic applications such as single-molecule Gunn oscillators. The device studied in this paper utilises $\mathrm{C}_{60}$ terminal groups attached to gold electrodes. These groups reduce the overall magnitude of the current, and therefore, for the future, it would be of interest to improve this proof of principle device. This could be accomplished by utilising alternative combinations of terminal groups and electrodes, which increase the current. One such possibility would be planar anchor groups on graphene electrodes, which are currently under development in a number of groups [50-52] and allow the imposition of an external electric field via a nearby gate.

\section{Supporting Information}

\section{Supporting Information File 1}

Additional theoretical information.

[http://www.beilstein-journals.org/bjnano/content/

supplementary/2190-4286-6-240-S1.pdf]

\section{Acknowledgement}

This work is supported by UK EPSRC grants EP/K001507/1, EP/J014753/1, EP/H035818/1 and the European Union MarieCurie Network MOLESCO.

\section{References}

1. Hiratsuka, Y.; Tada, T.; Oiwa, K.; Kanayama, T.; Uyeda, T. Q. P. Biophys. J. 2001, 81, 1555-1561. doi:10.1016/S0006-3495(01)75809-2

2. van den Heuvel, M. G.; Dekker, C. Science 2007, 317, 333-336. doi:10.1126/science.1139570

3. Fujimoto, K.; Kitamura, M.; Yokokawa, M.; Kanno, I.; Kotera, H.; Yokokawa, R. ACS Nano 2012, 7, 447-455. doi:10.1021/nn3045038

4. Aoyama, S.; Shimoike, M.; Hiratsuka, Y. Proc. Natl. Acad. Sci. U. S. A. 2013, 110, 16408-16413. doi:10.1073/pnas.1306281110

5. Taira, S.; Du, Y.-Z.; Hiratsuka, Y.; Uyeda, T. Q. P.; Yumoto, N.; Kodaka, M. Biotechnol. Bioeng. 2008, 99, 734-739. doi:10.1002/bit.21618

6. Song, W.; Möhwald, H.; Li, J. Biomaterials 2010, 31, 1287-1292. doi:10.1016/j.biomaterials.2009.10.026

7. Ionov, L.; Stamm, M.; Diez, S. Nano Lett. 2005, 5, 1910-1914. doi:10.1021/nl051235h

8. van den Heuvel, M. G. L.; de Graaff, M. P.; Dekker, C. Science 2006, 312, 910-914. doi:10.1126/science.1124258

9. Hess, H.; Clemmens, J.; Howard, J.; Vogel, V. Nano Lett. 2002, 2, 113-116. doi:10.1021/nl015647b

10. Bachand, G. D.; Hess, H.; Ratna, B.; Satir, P.; Vogel, V. Lab Chip 2009, 9, 1661-1666. doi:10.1039/B821055A

11. Fischer, T.; Agarwal, A.; Hess, H. Nat. Nanotechnol. 2009, 4, 162-166. doi:10.1038/nnano.2008.393

12. Schliwa, M.; Woehlke, G. Nature 2003, 422, 759-765. doi:10.1038/nature01601

13. Bruns, C. J.; Stoddart, J. F. Acc. Chem. Res. 2014, 47, 2186-2199. doi:10.1021/ar500138u 
14. Kumar, K. R. S.; Kamei, T.; Fukaminato, T.; Tamaoki, N. ACS Nano 2014, 8, 4157-4165. doi:10.1021/nn5010342

15. Gao, W.; Wang, J. Nanoscale 2014, 6, 10486-10494. doi:10.1039/C4NR03124E

16. Browne, W. R.; Feringa, B. L. Nat. Nanotechnol. 2006, 1, 25-35. doi:10.1038/nnano.2006.45

17. Balzani, V.; Credi, A.; Venturi, M. Molecular devices and machines: concepts and perspectives for the nanoworld; John Wiley \& Sons, Inc.: New York, NY, U.S.A., 2008.

18. Garcia-Garibay, M. A. Proc. Natl. Acad. Sci. U. S. A. 2005, 102, 10771-10776. doi:10.1073/pnas.0502816102

19. Karlen, S. D.; Garcia-Garibay, M. A. Amphidynamic crystals: Structural blueprints for molecular machines. Molecular Machines; Springer: Berlin, Germany, 2005; pp 179-227.

20. Davis, A. P. Angew. Chem., Int. Ed. 1998, 37, 909-910. doi:10.1002/(SICI)1521-3773(19980420)37:7<909::AID-ANIE909>3.0. $\mathrm{CO} ; 2-\mathrm{X}$

21. Kelly, T. R.; Sestelo, J. P.; Tellitu, I. J. Org. Chem. 1998, 63, 3655-3665. doi:10.1021/jo9723218

22. Hugel, T.; Holland, N. B.; Cattani, A.; Moroder, L.; Seitz, M.; Gaub, H. E. Science 2002, 296, 1103-1106. doi:10.1126/science.1069856

23. Liu, Y.; Flood, A. H.; Bonvallet, P. A.; Vignon, S. A.; Northrop, B. H.; Tseng, H.-R.; Jeppesen, J. O.; Huang, T. J.; Brough, B.; Baller, M.; Magonov, S.; Solares, S. D.; Goddard, W. A.; Ho, C.-M.; Stoddart, J. F. J. Am. Chem. Soc. 2005, 127, 9745-9759. doi:10.1021/ja051088p

24. Coskun, A.; Banaszak, M.; Astumian, R. D.; Stoddart, J. F.; Grzybowski, B. A. Chem. Soc. Rev. 2012, 41, 19-30. doi:10.1039/C1CS15262A

25. Bruns, C. J.; Stoddart, J. F. Nat. Nanotechnol. 2013, 8, 9-10. doi:10.1038/nnano.2012.239

26. Morin, J.-F.; Shirai, Y.; Tour, J. M. Org. Lett. 2006, 8, 1713-1716. doi:10.1021/ol060445d

27. Cumings, J.; Zettl, A. Science 2000, 289, 602-604. doi:10.1126/science.289.5479.602

28. Yu, M.-F.; Yakobson, B. I.; Ruoff, R. S. J. Phys. Chem. B 2000, 104, 8764-8767. doi:10.1021/jp002828d

29. Kolmogorov, A. N.; Crespi, V. H. Phys. Rev. Lett. 2000, 85, 4727-4730. doi:10.1103/PhysRevLett.85.4727

30.Zheng, Q.; Jiang, Q. Phys. Rev. Lett. 2002, 88, 045503. doi:10.1103/PhysRevLett.88.045503

31. Forró, L. Science 2000, 289, 560-561. doi:10.1126/science.289.5479.560

32. Williams, P. A.; Papadakis, S. J.; Patel, A. M.; Falvo, M. R.; Washburn, S.; Superfine, R. Phys. Rev. Lett. 2002, 89, 255502. doi:10.1103/PhysRevLett.89.255502

33. Rivera, J. L.; McCabe, C.; Cummings, P. T. Nano Lett. 2003, 3, 1001-1005. doi:10.1021/nl034171o

34. Sangtarash, S.; Sadeghi, H.; Ahmadi, M. T.; Ghadiry, M. H.; Anwar, S.; Ismail, R. J. Comput. Theor. Nanosci. 2012, 9, 1554-1557. doi:10.1166/jctn.2012.2243

35. Grace, I. M.; Bailey, S. W.; Lambert, C. J. Phys. Rev. B 2004, 70, 153405. doi:10.1103/PhysRevB.70.153405

36. Bailey, S. W. D.; Amanatidis, I.; Lambert, C. J. Phys. Rev. Lett. 2008, 100, 256802. doi:10.1103/PhysRevLett.100.256802

37. Cai, K.; Yin, H.; Qin, Q. H.; Li, Y. Nano Lett. 2014, 14, 2558-2562. doi: $10.1021 / \mathrm{nl} 5003608$

38. Fennimore, A. M.; Yuzvinsky, T. D.; Han, W.-Q.; Fuhrer, M. S.; Cumings, J.; Zettl, A. Nature 2003, 424, 408-410. doi:10.1038/nature01823
39. Bourlon, B.; Glattli, D. C.; Miko, C.; Forró, L.; Bachtold, A. Nano Lett. 2004, 4, 709-712. doi:10.1021/nl035217g

40. Niguès, A.; Siria, A.; Vincent, P.; Poncharal, P.; Bocquet, L. Nat. Mater. 2014, 13, 688-693. doi:10.1038/nmat3985

41. Leary, E.; González, M. T.; van der Pol, C.; Bryce, M. R.; Filippone, S.; Martín, N.; Rubio-Bollinger, G.; Agraït, N. Nano Lett. 2011, 11, 2236-2241. doi:10.1021/nl200294s

42. Soler, J. M.; Artacho, E.; Gale, J. D.; García, A.; Junquera, J.; Ordejón, P.; Sánchez-Portal, D. J. Phys.: Condens. Matter 2002, 14, 2745. doi:10.1088/0953-8984/14/11/302

43. Dion, M.; Rydberg, H.; Schröder, E.; Langreth, D. C.; Lundqvist, B. I. Phys. Rev. Lett. 2004, 92, 246401. doi:10.1103/PhysRevLett.92.246401

44. Langreth, D. C.; Dion, M.; Rydberg, H.; Schröder, E.; Hyldgaard, P.; Lundqvist, B. I. Int. J. Quantum Chem. 2005, 101, 599-610. doi:10.1002/qua.20315

45. Landauer, R. Z. Phys. B: Condens. Matter 1987, 68, 217-228. doi:10.1007/BF01304229

46. Ferrer, J.; Lambert, C. J.; García-Suárez, V. M.; Manrique, D. Zs.; Visontai, D.; Oroszlany, L.; Rodríguez-Ferradás, R.; Grace, I.; Bailey, S. W. D.; Gillemot, K.; Sadeghi, H.; Algharagholy, L. A. New J. Phys. 2014, 16, 093029. doi:10.1088/1367-2630/16/9/093029

47. Sadeghi, H.; Mol, J. A.; Lau, C. S.; Briggs, G. A. D.; Warner, J.; Lambert, C. J. Proc. Natl. Acad. Sci. U. S. A. 2015, 112, 2658-2663. doi:10.1073/pnas.1418632112

48. Lambert, C. Chem. Soc. Rev. 2015, 44, 875-888. doi:10.1039/c4cs00203b

49. Martín, S.; Manrique, D. Zs.; García-Suárez, V. M.; Haiss, W.; Higgins, S. J.; Lambert, C. J.; Nichols, R. J. Nanotechnology 2009, 20, 125203. doi:10.1088/0957-4484/20/12/125203

50. Sadeghi, H.; Sangtarash, S.; Lambert, C. J. Beilstein J. Nanotechnol. 2015, 6, 1413. doi:10.3762/bjnano.6.146

51. Mol, J. A.; Lau, C. S.; Lewis, W. J. M.; Sadeghi, H.; Roche, C.; Cnossen, A.; Warner, J. H.; Lambert, C. J.; Anderson, H. L.; Briggs, G. A. D. Nanoscale 2015, 7, 13181-13185. doi:10.1039/C5NR03294F

52. Prins, F.; Barreiro, A.; Ruitenberg, J. W.; Seldenthuis, J. S.; Aliaga-Alcalde, N.; Vandersypen, L. M. K.; van der Zant, H. S. J. Nano Lett. 2011, 11, 4607-4611. doi:10.1021/nl202065x

\section{License and Terms}

This is an Open Access article under the terms of the Creative Commons Attribution License (http://creativecommons.org/licenses/by/2.0), which permits unrestricted use, distribution, and reproduction in any medium, provided the original work is properly cited.

The license is subject to the Beilstein Journal of Nanotechnology terms and conditions: (http://www.beilstein-journals.org/bjnano)

The definitive version of this article is the electronic one which can be found at:

doi:10.3762/bjnano.6.240 